\title{
High-affinity tamoxifen analogues retain extensive positional disorder when bound to calmodulin
}

\author{
Lilia Milanesi ${ }^{1,2}$, Clare R. Trevitt ${ }^{1}$, Brian Whitehead ${ }^{1}$, Andrea M. Hounslow ${ }^{1}$, Salvador Tomas ${ }^{2,6}$, \\ Laszlo L. P. Hosszu ${ }^{1,3}$, Christopher A. Hunter ${ }^{4}$, and Jonathan P. Waltho ${ }^{1,5}$ \\ ${ }^{1}$ Department of Molecular Biology and Biotechnology, University of Sheffield, Sheffield S10 2TN, UK \\ ${ }^{2}$ Department of Biological Sciences, School of Science, Birkbeck University of London, \\ London WC1E 7HX, UK \\ ${ }^{3}$ Medical Research Council Prion Unit, University College of London Institute of Neurology, \\ Queen Square, London WCN1 3BG, UK \\ ${ }^{4}$ Department of Chemistry, University of Cambridge, Lensfield Road, Cambridge CB2 1EW, UK \\ ${ }^{5}$ Manchester Institute of Biotechnology, University of Manchester, 131 Princess Street, \\ Manchester M1 7DN, UK \\ ${ }^{6}$ Departament de Química, Universitat de les Illes Balears, Cra. de Valldemossa, \\ km 7.5. 07122 Palma de Mallorca, Spain
}

Correspondence: Jonathan P. Waltho (j.waltho@sheffield.ac.uk)

Received: 14 January 2021 - Discussion started: 5 February 2021

Revised: 29 March 2021 - Accepted: 3 April 2021 - Published: 13 August 2021

\begin{abstract}
Using a combination of NMR and fluorescence measurements, we have investigated the structure and dynamics of the complexes formed between calcium-loaded calmodulin $(\mathrm{CaM})$ and the potent breast cancer inhibitor idoxifene, a derivative of tamoxifen. High-affinity binding $\left(K_{d} \sim 300 \mathrm{nM}\right)$ saturates with a $2: 1$ idoxifene : CaM complex. The complex is an ensemble where each idoxifene molecule is predominantly in the vicinity of one of the two hydrophobic patches of CaM but, in contrast with the lower-affinity antagonists TFP, $\mathrm{J}-8$, and W-7, does not substantially occupy the hydrophobic pocket. At least four idoxifene orientations per domain of CaM are necessary to satisfy the intermolecular nuclear Overhauser effect (NOE) restraints, and this requires that the idoxifene molecules switch rapidly between positions. The $\mathrm{CaM}$ molecule is predominantly in the form where the $\mathrm{N}$ and $\mathrm{C}$-terminal domains are in close proximity, allowing for the idoxifene molecules to contact both domains simultaneously. Hence, the $2: 1$ idoxifene : CaM complex illustrates how high-affinity binding occurs without the loss of extensive positional dynamics.
\end{abstract}

\section{Introduction}

Calmodulin $(\mathrm{CaM})$ is an important intracellular calcium receptor found in all eukaryotic cells. Calcium-loaded CaM binds to more than 300 target enzymes that modulate various cellular functions (Ikura and Ames, 2006; Swulius and Waxham, 2008). CaM consists of two globular domains separated by a solvent-exposed helical region that is not continuous in solution (the so-called flexible tether), allowing the two domains to be independently mobile (Barbato et al., 1992; Chou et al., 2001; Trevitt et al., 2005). On binding calcium, the four helices in each of the two globular domains undergo a large conformational change, where the domains become less compact and a hydrophobic pocket is opened (Finn et al., 1995; Kuboniwa et al., 1995; Zhang et al., 1995). These hydrophobic pockets play a central role in the binding of various CaM targets (Meador et al., 1992; Ikura et al., 1992; Craven et al., 1996; Osawa et al., 1998; Harmat et al., 2000; Kovesi et al., 2008).

The proposed mechanism of action of CaM-mediated enzyme regulation is based largely on structural studies of complexes between $\mathrm{CaM}$ and peptides of 20-30 residues corresponding to $\mathrm{CaM}$ interaction domains rather than intact en- 
zymes. On binding of most of these peptides to $\mathrm{CaM}$, the flexible tether between the two globular domains bends such that the $\mathrm{N}$-terminal domain comes close to the $\mathrm{C}$-terminal domain and the $\alpha$ helices that usually form in the bound peptides stabilize and fix the position of the two CaM domains (Maximciuc et al., 2006; Frederick et al., 2007). In this mode of binding, also called the wrap-around mode, the hydrophobic pockets in the globular domains become occupied by side chains of hydrophobic residues within the peptides and the complex adopts a compact, globular structure. However, more recent structural studies show alternative modes of $\mathrm{CaM}$ binding to proteins and peptides. In some of these complexes, $\mathrm{CaM}$ adopts an extended structure more similar to that of uncomplexed CaM (Elshorst et al., 1999; Samal et al., 2011), and the hydrophobic pockets of CaM do not bind to the hydrophobic residues of the target peptide, although nanomolar binding affinity is retained (Yamauchi et al., 2003; Izumi et al., 2008).

A similar diversity of binding modes has been observed in $\mathrm{CaM}$ bound to small-molecule antagonists that share features of the target peptides. They have hydrophobic regions and basic functional groups but have greater mobility in the CaMbound state, making it more difficult to determine the extent of domain closure in these complexes relative to those with peptides (Prozialeck and Weiss, 1982; Craven et al., 1996; Osawa et al., 1998). Some of these antagonists, such as the antipsychotic drug trifluoperazine (TFP), the highly selective inhibitors of CaM-mediated processes $\mathrm{W}-7$ and J-8, calmidazolium, the arylalkylamine derivative DPD, and certain bifunctional ligands, bind $\mathrm{CaM}$ with affinities in the nanomolar to low micromolar range. These ligands form complexes that are often characterized by a higher degree of proximity of the two CaM domains compared with the complexes between the protein and low-affinity ligands (Reid et al., 1990; Osawa et al., 1998, 1999; Trevitt et al., 2005; Kovesi et al., 2008). In contrast, an alternative mode of binding has also been reported for the high-affinity antagonist Kar-2 that does not involve the hydrophobic pockets of CaM (Horvath et al., 2005).

In the present study we show that the complex between $\mathrm{CaM}$ and the high-affinity antagonist idoxifene represents a still different binding mode of a CaM antagonist. Idoxifene is a triphenylethylene-derivative analogue of tamoxifen (Fig. 1), one of the first agents of choice for the treatment and prevention of breast cancer (Marshall, 1998; Powles, 2013). The classical view of the mechanism of action of tamoxifen is that it competes with estradiol for binding to the estrogen receptor (ER) (Shiau et al., 1998). However, several studies indicate that tamoxifen inhibition of breast cancer growth is the result of a complex interplay involving both ER binding and CaM antagonism (Gulino et al., 1986; Cifuentes et al., 2004; Li and Sacks, 2007; Gallo et al., 2008). In addition to its therapeutic efficacy for breast cancer, tamoxifen also has antifungal activity and inhibits the growth of various tumours by a complex mechanism that requires $\mathrm{CaM}$

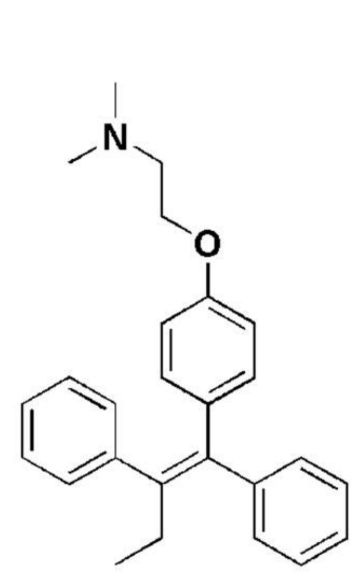

Tamoxifen

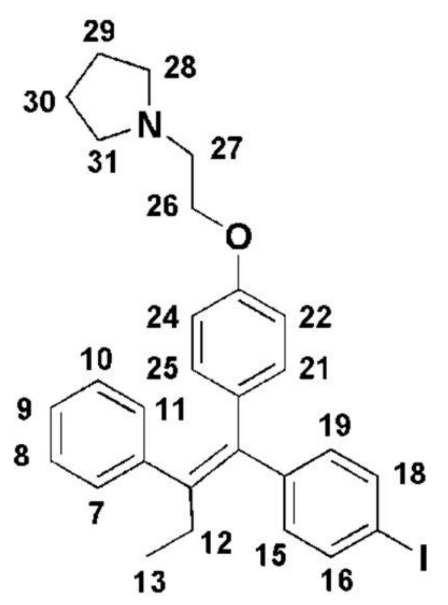

Idoxifene
Figure 1. The chemical structures of tamoxifen and idoxifene. The numbering scheme for idoxifene used here is shown: phenyl group: H7-H11; ethyl group: H12-H13; p-iodo-phenyl group: H15-H19; p-phenoxy group: $\mathrm{H} 21-\mathrm{H} 25$; pyrrolidine group: $\mathrm{H} 28-\mathrm{H} 31$.

antagonism in all cases (Cifuentes et al., 2004; Dolan et al., 2009; Pawar et al., 2009; Byer et al., 2011). Hence, an understanding of the structural determinants of tamoxifen binding to CaM may help in the development of yet more effective therapies. To date there have been few such studies: insights into the nature of the complex come mainly from molecular modelling and structure activity relationship studies (SARs) (Edwards et al., 1992; Hardcastle et al., 1995, 1996). These studies led to the synthesis of idoxifene, a derivative of tamoxifen in which the basic dimethyl amino side chain has been replaced by a pyrrolidine and iodine has been placed in one of the phenyl rings (Fig. 1). In vitro, idoxifene showed an inhibition potency for CaM of some 4-5 times that of tamoxifen, a higher toxicity towards ER-positive MCF-7 human breast cancer cells, and higher in vivo clinical activity compared to tamoxifen (MacGregor and Jordan, 1998; Dowsett et al., 2000). These properties of idoxifene make it an interesting target for a structural study of its binding to CaM. Here we use heteronuclear multidimensional NMR and fluorescence spectroscopy to determine the binding affinity, stoichiometry, and solution-binding mode of idoxifene to $\mathrm{Ca}^{2+}$ $\mathrm{CaM}$. A comparison with a previously determined molecular model of the same complex and the structures of CaM peptides and CaM-ligand complexes reveals an unusual binding mode that broadens the repertoire of recognition processes involving CaM.

\section{Materials and methods}

\subsection{Sample preparation}

Idoxifene was a gift from the CRC Institute of Cancer Research at Sutton, Surrey, UK. Unlabelled and uniformly 
${ }^{13} \mathrm{C} /{ }^{15} \mathrm{~N}$-labelled recombinant mammalian CaM were prepared as described previously (Vogel et al., 1983). Purified, lyophilized, calcium-free protein was dissolved to a concentration of $3 \mathrm{mM}$ in $50 \mathrm{mM} \mathrm{KCl}^{2} 5 \mathrm{mM} \mathrm{NaN}_{3}$, and $10 \%$ $\mathrm{D}_{2} \mathrm{O}$. 3-(trimethylsilyl) propionic-2,2,3,3,- $d_{4}$ acid, sodium salt (TSP, $0.1 \mathrm{mM}$ ), was added as a reference compound. Appropriate quantities of $\mathrm{CaCl}_{2}$ solution $(6 \mathrm{~mol}$ per mole of $\mathrm{CaM}$ ) were added to yield calcium-saturated protein and checked by observing diagnostic amide chemical shift changes in the $1 \mathrm{D}{ }^{1} \mathrm{H}$ NMR spectrum. The final sample volume was $450 \mu \mathrm{L}$. The $\mathrm{pH}$ of the solution was adjusted to 6.0 (uncorrected value) by adding microlitre quantities of $0.1 \mathrm{mM} \mathrm{HCl}$ and $\mathrm{NaOH}$. CaM samples were otherwise unbuffered because the apo-protein has sufficient buffering capacity at $\mathrm{pH} 6.0$ and the extrinsic buffers available without non-exchangeable protons compete for calcium binding. The $\mathrm{KCl}$ concentration used here mimics conditions in which the calcium affinity of CaM was determined (Linse et al., 1991) and was chosen to minimize the likelihood of idoxifene precipitation at the end of the titration when excess ligand is present. This unbuffered system and salt concentration are in keeping with previous studies of CaM $(50-100 \mathrm{mM} \mathrm{KCl})$ (Finn et al., 1995; Craven et al., 1996; Trevitt et al., 2005).

Stock solutions of idoxifene were prepared at a concentration of $60 \mathrm{mM}$ in $\mathrm{CD}_{3} \mathrm{OD}$. Idoxifene was added to the $\mathrm{CaM}$ solution up to a maximum ligand : protein molar ratio of $2.4: 1 .{ }^{1} \mathrm{H}$ and $2 \mathrm{D}^{1} \mathrm{H}_{-}{ }^{15} \mathrm{~N}$ HSQC spectra were recorded on the protein solutions and, for each successive addition of idoxifene, in steps of 0.2 equivalents of idoxifene to CaM. A control titration with $\mathrm{CD}_{3} \mathrm{OD}$ was carried out under the same conditions to correct for the small effects of $\mathrm{CD}_{3} \mathrm{OD}$ on the protein chemical shifts. The $\mathrm{pH}$ of the protein solutions was monitored throughout the titrations, and when necessary small additions of acid or base were made to maintain the $\mathrm{pH}$ at 6.0.

\subsection{Fluorescence spectroscopy}

Binding of $\mathrm{CaM}$ to idoxifene was monitored using changes in the intrinsic fluorescence of idoxifene at $293 \mathrm{~K}$. Titrations were performed using a Cary Eclipse fluorescence spectrophotometer, with excitation at $295 \mathrm{~nm}$ and observation of emission at $450 \mathrm{~nm}$. Idoxifene and $\mathrm{CaM}$ solutions were prepared in $45 \mathrm{mM} \mathrm{KCl}$ and $9 \mathrm{mM} \mathrm{CaCl}_{2}$, $\mathrm{pH} 6.0$ with $10 \%$ methanol, and $\mathrm{CaM}$ was added to idoxifene in aliquots of 0.1 molar equivalents. Titrations were performed with 10 and $0.54 \mu \mathrm{M}$ idoxifene, using $90 \mu \mathrm{M}$ and $4.5 \mu \mathrm{MCaM}$ respectively. Concentrations were verified by quantitative 1D ${ }^{1} \mathrm{H}$ NMR relative to TSP for idoxifene and by absorbance at $276 \mathrm{~nm}$ for $\mathrm{CaM}$, using the extinction coefficient $3300 \mathrm{~cm}^{-1} \mathrm{M}^{-1}$. The $0.54 \mu \mathrm{M}$ idoxifene titration was fitted for $K_{d}$ using a $2: 1$ idoxifene : CaM stoichiometry and independently fitted for both $K_{d}$ and stoichiometry using inhouse software.

\subsection{NMR spectroscopy}

NMR experiments were carried out at $310 \mathrm{~K}$. The $\mathrm{H}_{2} \mathrm{O}$ resonance was suppressed by on-resonance low-power presaturation (typically applying a $10 \mathrm{~Hz}$ field for $800 \mathrm{~ms}$ ) during the relaxation delay, followed by a SCUBA sequence employing two composite $\Pi$ pulses separated by $30 \mathrm{~ms}$ delay (Brown et al., 1988). The data were acquired using standard heteronuclear NMR experiments, processed using the program FELIX, and deconvoluted as described previously (Craven et al., 1996). Proton, carbon, and nitrogen frequencies were referenced relative to TSP using values of 0.251449530 and 0.101329118 for $\gamma_{\mathrm{C}} / \gamma_{\mathrm{H}}$ and $\gamma_{\mathrm{N}} / \gamma_{\mathrm{H}}$ respectively (Wishart et al., 1995).

\subsubsection{Resonance and NOE assignment}

Resonance assignment of free CaM was based on the assignment of Drosophila CaM (BMRB entry 547) and it was verified using TOCSY and NOESY-HSQC experiments (Craven et al., 1996). For the idoxifene : CaM complex, the amide ${ }^{1} \mathrm{H}^{15} \mathrm{~N}$ correlations were followed in the titration series of HSQC spectra using 3D TOCSY-HSQC and NOESY-HSQC experiments. The assignments of backbone and side-chain resonances of ${ }^{13} \mathrm{C},{ }^{15} \mathrm{~N}$-labelled $\mathrm{CaM}$ in the $2: 1$ idoxifene : $\mathrm{CaM}$ complex were confirmed using previously described protocols (Craven et al., 1996; Osawa et al., 1998), including $3 \mathrm{D}{ }^{13} \mathrm{C}$-edited NOESY-HSQC and 3D long-range ${ }^{13} \mathrm{C}-{ }^{13} \mathrm{C}$ correlation experiments for the assignment of the $\varepsilon$ methyl resonances of methionine. The assignment of idoxifene in the absence of protein (Fig. S1) was carried out in $\mathrm{D}_{2} \mathrm{O}$ at pH 3.0, as the ligand is insoluble at $\mathrm{pH}$ 6.0. Idoxifene resonances in the complex were assigned using $2 \mathrm{D}$ TOCSY and a $2 \mathrm{D}{ }^{13} \mathrm{C}$ ${ }^{15} \mathrm{~N}$-double-half-filtered NOESY experiment acquired with a mixing time of $100 \mathrm{~ms}$, in line with previous solution structures of CaM-small ligand complexes (Craven et al., 1996; Osawa et al., 1998). The identity of resonances involved in intermolecular NOEs in the complex was also discerned using the $2 \mathrm{D}{ }^{13} \mathrm{C}-{ }^{15} \mathrm{~N}$-double-half-filtered NOESY experiment and, for protein resonances, confirmed using a $3 \mathrm{D} \omega_{1}{ }^{13} \mathrm{C}$ ${ }^{15} \mathrm{~N}$-half-filtered ${ }^{13} \mathrm{C}$-edited NOESY-HSQC spectrum with the same mixing time. For matching to the assignment data, tolerances of 0.03 and $0.3 \mathrm{ppm}$ were used for ${ }^{1} \mathrm{H}$ and ${ }^{13} \mathrm{C}$ frequencies respectively. The chemical shift values of the peak centres were converted to X-PLOR restraints using in-house software. For the idoxifene: CaM complex, 180 ligand-protein NOEs were observed, of which 110 were unambiguously assigned.

\subsubsection{Line-shape analysis}

Line shapes were calculated for a simple two-state exchange model using standard equations (McConnell, 1958). The transverse relaxation times were adjusted to match the observed line width, and the value of the off-rate was varied 
to optimize the agreement between the calculated and experimental data. A normalizing factor was applied to all data to correct for the constant intensity loss observed throughout the titration as a result of dilution. This was calculated by determining the mean intensity loss of a number of peaks for which no change in chemical shifts was seen on binding idoxifene and hence were unaffected by the exchange processes. In interpreting the line-shape analysis in terms of absolute stoichiometry, it is imperative to be certain of the ligand and protein concentrations used. For the protein this was initially determined using UV absorbance and for the ligand using dry weight. The final concentration in the $2: 1$ complexes was then checked by comparison of peaks in the 1D spectrum, which confirmed that the concentrations were correct to within $10 \%$.

\subsection{Structure calculations}

Restraints were classified as strong, medium, or weak and were assigned upper bounds of (i) 2.5, 3.5, and 5.0 $\AA$, (ii) 3.0, 4.0, and 5.0 $\AA$, or (iii) all as $5.0 \AA$ in separate calculations to accommodate the inherent uncertainty involved in converting cross-peak intensities to more precise distances when there is the possibility of conformational exchange of the ligand in its binding site. All NOE restraints were introduced using $1 / r^{6}$ sum averaging to accommodate reorientation of aromatic rings and isopropyl groups via bond rotation. Structures were calculated with X-PLOR 3.1 using a molecular dynamics simulated annealing protocol for conventional protein structure determination (Brünger, 1992; Nilges, 1995). For the first 3 ps, a high temperature (1500 K) was maintained, and the weight on the core repulsion potential energy term was kept very low. This was followed by an 18 ps cooling stage in which the temperature was reduced in $50 \mathrm{~K}$ steps, and the weight on the core repulsion term was gradually increased. Finally, the structures were subjected to 250 steps of conjugate gradient energy minimization. As a refinement stage, the temperature was increased to $1500 \mathrm{~K}$ and the above cycle repeated. During the high-temperature stage of the protocol, a square-well NOE potential was used, with harmonic sides. During the second part of the protocol, the X-PLOR soft-square potential was used, which smoothly changes the harmonic potential to a linear potential for large restraint violations. The energy constant for the harmonic potential was $5.0 \mathrm{kcal} \mathrm{mol}^{-1} \AA^{-2}$. The slope of the asymptote was $0.5 \mathrm{kcal} \mathrm{mol}^{-1} \AA^{-1}$. The switching region between the two regimes was approximately between 0.5 and $2 \AA$ above the upper restraint bound. The parallhdg.pro parameter set of X-PLOR was used, with the X-PLOR quartic repel potential to represent the repulsive part on the interatomic interactions. No attractive or electrostatic terms were used. The final weight on the repulsive term was $4 \mathrm{kcal} \mathrm{mol}^{-1} \AA^{-4}$.

For ab initio structure calculations, extended protein coordinates with random initial velocities were used as a seed, and two idoxifene molecules were placed at random within a box of side $60 \AA$ centred on the centre of mass of CaM. For NOE-restrained docking calculations, protein coordinates were taken from either the $2.4 \AA$ resolution structure of $\mathrm{CaM}$ in a complex with a myosin light-chain kinase peptide (pdb - Protein Data Bank - entry 1cdl, Meador et al., 1992) or the tr2c domain of the $1.7 \AA$ resolution X-ray structure of mammalian CaM (pdb entry 1cll, Chattopadhyaya et al., 1992). Two sets of starting positions of the idoxifene molecules were investigated - molecules placed at random in a box and molecules manually docked into the hydrophobic pocket - to exclude any bias away from occupancy of the hydrophobic pocket through restrictions in the sampling of the relative positions of molecules. For the former, a fresh idoxifene starting conformation for each calculation was created by first transforming the coordinates in an extended conformation by a random rigid body rotation. The centre of mass was then placed at random within a box of side $60 \AA$ centred on the centre of mass of the protein molecule. The idoxifene molecule was treated as flexible, subject to restraints of covalent geometry and van der Waals contacts. The side chains involved in intermolecular NOEs were either fixed with the remainder of the protein or allowed the same internal flexibility as the idoxifene molecules, and the two calculations were compared. For the manual docking of idoxifene molecules into the hydrophobic pocket of the protein, 50 structures were generated in which different hydrophobic parts of the idoxifene molecule were placed deep inside the hydrophobic pocket of the domain, and the molecules were subjected to 250 steps of conjugate gradient energy minimization to remove steric clashes. In each case part of the idoxifene molecule remained within the hydrophobic pocket. These structures were then used as starting coordinates for the structure calculation protocols described above. For both sets of starting positions, the final distribution of structures was indistinguishable. When more than one idoxifene molecule per domain of CaM was included in the calculation, the core repulsion terms between idoxifene molecules was set to zero, whereas those within each idoxifene and between each idoxifene and the protein were increased during the course of the calculation as described above. This meant that there was no energy penalty to atoms from more than one idoxifene molecule occupying the same space. An NOE restraint was satisfied by the proximity to the protein atoms of atoms from any individual idoxifene molecule.

\section{Results}

\subsection{Titration of CaM with idoxifene}

The binding stoichiometry and affinity between idoxifene and $\mathrm{CaM}$ were measured using changes in idoxifene fluorescence at $450 \mathrm{~nm}$ upon addition of increasing amounts of $\mathrm{Ca}^{2+}$-CaM. Using $10 \mu \mathrm{M}$ idoxifene, the response on $\mathrm{CaM}$ addition was linear up to a stoichiometry of $2: 1$ idoxifene : CaM (Fig. 2a). Using $540 \mathrm{nM}$ idoxifene (Fig. 2b), the data 
fit to a $K_{d}$ of $340 \pm 30 \mathrm{nM}$ using a stoichiometry of $2: 1$ idoxifene : CaM. When both $K_{d}$ and stoichiometry were allowed to vary, the data best fit to a $K_{d}$ of $180 \pm 50 \mathrm{nM}$ and a stoichiometry of $1.7: 1$ idoxifene : $\mathrm{CaM}$, but there is covariance between these parameters in the range that includes a 2: 1 stoichiometry and a $K_{d}$ of $340 \mathrm{nM}$. A chi ${ }^{2}$ analysis of fits indicated a clear minimum at $K_{d}=300 \mathrm{nM}$. The binding of idoxifene to $\mathrm{CaM}$ was monitored independently using $1 \mathrm{D}{ }^{1} \mathrm{H}$ NMR, and the perturbations of low field amide ${ }^{1} \mathrm{H}$ resonances during the addition of up to 2.4 equivalents of idoxifene are shown in Fig. 2c. Where the chemical shift changes induced by complex formation are much greater than $0.05 \mathrm{ppm}$, two sets of resonances are detected during the titration (e.g. I27, I100, and G134 in Fig. 2c). Thus, for these resonances, the dissociation rate of the complex is predominantly in the slow exchange regime on the NMR timescale. The resonances corresponding to free CaM disappeared when two equivalents of ligand were added, in line with the stoichiometry of $2: 1$ reported by the fluorescence measurements using $10 \mu \mathrm{M}$ idoxifene. Some resonances experienced a reduction of peak height at intermediate ligand concentrations and re-sharpened when two equivalents of idoxifene were added (e.g. D64 and N137 in Fig. 2c). The extent of line broadening allowed an estimate for the off-rate of $30 \pm 10 \mathrm{~s}^{-1}$ to be obtained, which, in combination with the measured $K_{d}$ value, indicates that the complex forms with a diffusion-controlled on-rate of ca. $1 \times 10^{8} \mathrm{M}^{-1} \mathrm{~s}^{-1}$. D64 and N137 occupy equivalent positions in the CaM structure, being located in position 9 of $\mathrm{Ca}^{2+}$ binding loops II and IV, and contribute to the three residue $\beta$ strands in each domain. The resonances of T28 and S101, the equivalent residues in loops I and III, show slow exchange behaviour, as do all of the other residues in the short $\beta$ sheets of CaM (e.g. I27 and I100 in Fig. 2c). The anomalous behaviour of D64 and N137 may result from a sensitivity of these residues to the occupation of the other globular domain of CaM by idoxifene.

\subsection{Chemical shift changes}

The NMR resonances of free idoxifene were assigned on the basis of characteristic chemical shifts of model compounds (2-pyrrolidinoethanol, iodo-benzene, methoxybenzene), NOEs, and correlations observed in a $2 \mathrm{D}{ }^{1} \mathrm{H}$ TOCSY spectrum (Table 1 and Fig. S1). The backbone and side-chain resonances of ${ }^{13} \mathrm{C},{ }^{15} \mathrm{~N}$-labelled $\mathrm{CaM}$ in the $2: 1$ idoxifene : CaM complex were assigned using previously described protocols (Craven et al., 1996; Osawa et al., 1998). The acquisition of intra- and inter-residue carbonyl shifts was essential in order to distinguish residues with degenerate $\mathrm{C} \alpha$ shifts, such as D50 and D122. More severe overlap present in the aromatic region of the spectra of the complex prevented the assignment of the $\zeta$ resonances of the phenylalanine residues. The assignment of the $\varepsilon$ methyl resonances of methionine residues was achieved using a combination of 3D long-range ${ }^{13} \mathrm{C}^{-13} \mathrm{C}$ correlation (LRCC) and $3 \mathrm{D}{ }^{13} \mathrm{C}$ -
Table 1. ${ }^{1} \mathrm{H}$ chemical shifts of idoxifene, free in solution and bound to $\mathrm{CaM}$.

\begin{tabular}{rrrr}
\hline Resonance & Free $^{\mathrm{a}}$ & Bound $^{\mathrm{b}}$ & \\
\hline${ }^{1} \mathrm{H}$ & $\delta$ & $\delta$ & $\Delta \delta$ \\
\hline $16 / 18$ & 7.52 & 7.42 & -0.10 \\
$15 / 19$ & 6.86 & 6.84 & -0.02 \\
$21 / 25$ & 6.69 & 6.71 & +0.02 \\
$22 / 24$ & 6.47 & 6.55 & +0.08 \\
$7 / 11$ & 6.99 & 6.98 & -0.01 \\
$8,9,10^{\mathrm{c}}$ & 7.06 & 7.08 & +0.02 \\
12 & 2.23 & 2.31 & +0.08 \\
13 & 0.73 & 0.78 & +0.05 \\
26 & 3.96 & 4.10 & +0.14 \\
27 & 3.43 & 3.50 & +0.07 \\
$28 / 31$ & $3.39 / 3.06$ & 3.30 & +0.07 \\
$29 / 30$ & 1.96 & 1.99 & +0.03 \\
\hline
\end{tabular}

${ }^{\mathrm{a}} \mathrm{D}_{2} \mathrm{O}, \mathrm{pH}$ 3.0. ${ }^{\mathrm{b}} \mathrm{D}_{2} \mathrm{O}, \mathrm{pH}$ 6.0. ${ }^{\mathrm{c}}$ Unresolved resonances.

edited NOESY-HSQC experiments (seven of the nine were assigned). Some resonances from residues L69-K77 were attenuated, particularly their $\mathrm{C} \alpha$ resonances, indicative of conformational exchange in the flexible tether region between domains in the complex.

A summary of the backbone chemical shift changes observed on binding of idoxifene to $\mathrm{CaM}$ is shown in Fig. 3 as a weighted average (WA) of the changes in all five backbone resonances, obtained using the equation

$|\mathrm{WA}| \cong \sum\left|\delta_{\mathrm{CaM}: \text { drug }}-\delta_{\mathrm{CaM}}\right| /\left|\Delta_{\max }\right|$,

where the summation extends over the backbone atoms, $\delta_{\mathrm{CaM} \text { drug }}$ and $\delta_{\mathrm{CaM}}$ are the chemical shifts observed in the complex and in free $\mathrm{CaM}$, and $\Delta_{\max }$ is the largest chemical shift change observed for each type of nucleus. The chemical shift changes in each of the five backbone atoms are shown in Fig. S2.

Substantial chemical shift changes are seen in many contiguous stretches of the backbone such as E67-I85 and F141T146 and are mapped onto the open structure of $\mathrm{CaM}$ in Fig. 4a. In contrast, some residues such as V55 display large shift changes, whereas surrounding residues are hardly perturbed. Residues E67-I85 include the flexible tether between the two domains, and the chemical shift changes here are closely comparable with those in the same region observed on formation of the CaM : M13 complex (Ikura et al., 1991), where $\mathrm{CaM}$ wraps its two domains around a helical peptide in a compact, globular structure.

The positions of the side-chain chemical shift changes observed on binding of idoxifene to $\mathrm{CaM}$ are shown in Fig. $4 \mathrm{~b}$. The majority of protein side-chain resonances move by less than $0.05 \mathrm{ppm}$ for ${ }^{1} \mathrm{H}$ and $0.3 \mathrm{ppm}$ for ${ }^{13} \mathrm{C}$. The larger sidechain shift changes are confined to residues around the hydrophobic pockets of both $\mathrm{CaM}$ domains, with the largest 
(a)

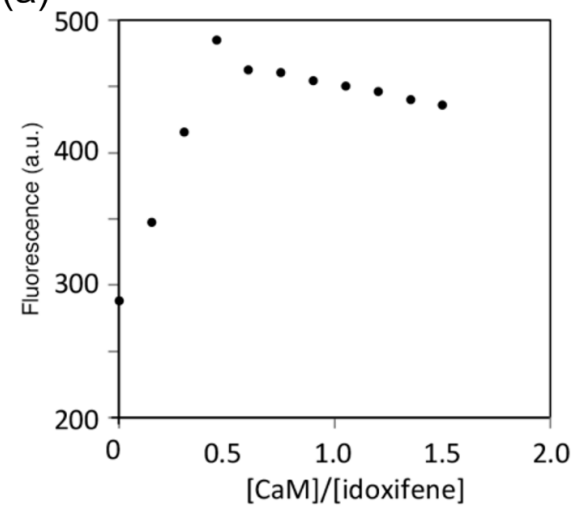

(b)

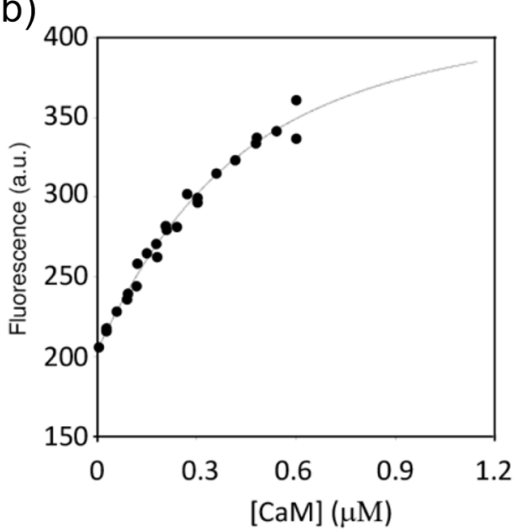

(c)

(c) G25, G61

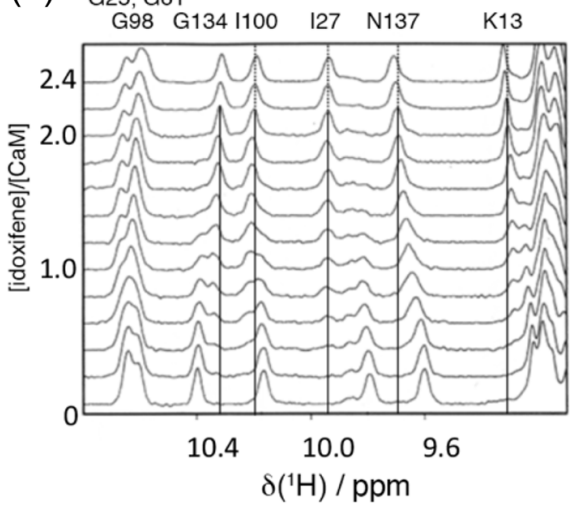

Figure 2. Titration of idoxifene with calmodulin. (a) The changes in fluorescence intensity of $10 \mu \mathrm{M}$ idoxifene at $450 \mathrm{~nm}$ on addition of CaM up to $15 \mu \mathrm{M}$. Saturation is reached at a concentration ratio of CaM to idoxifene of 0.5 , indicating the presence of two binding sites with submicromolar binding affinity. (b) Repeat of (a) but with $0.54 \mu \mathrm{M}$ idoxifene and increasing CaM concentrations from $0.02 \mu \mathrm{M}$ up to $0.6 \mu \mathrm{M}$. The data were fitted to a binding isotherm with a $2: 1$ idoxifene : CaM binding stoichiometry. (c) A region of the $1 \mathrm{D}{ }^{1} \mathrm{H}$ spectra acquired during the titration of idoxifene into $3 \mathrm{mM} \mathrm{CaM}$. Solid lines highlight the completion of the slow exchange event for the assigned resonances at $2: 1$ idoxifene : CaM. Dotted lines are drawn for resonances undergoing shift changes beyond the end point of the titration. G134, I100, and $\mathrm{I} 27$ are representative of the slow exchange regime observed for CaM resonances during the titration, while N137 is representative of an intermediate exchange character.

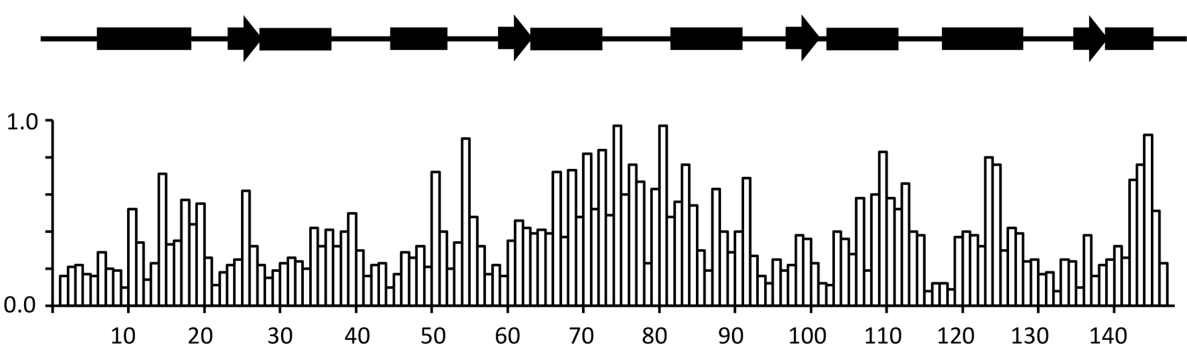

Figure 3. Chemical shift changes for backbone CaM resonances upon binding idoxifene. Normalized, weighted average chemical shift changes are plotted against the primary sequence of CaM. Secondary structure elements are indicated above the histogram as solid boxes for $\alpha$ helices, solid arrows for $\beta$ strands, and thin lines for unstructured regions. The flexible tether (E67-V85) experiences the largest chemical shift changes upon complex formation.

changes observed for methionine residues, in particular the $\beta$ and $\varepsilon$ resonances of M71 and M144 (Fig. 4c), which occupy equivalent positions near the $\mathrm{C}$ terminus of each domain, on the rim of their hydrophobic pockets. Also of note are the chemical shift changes in phenylalanine ring resonances. Only F68 and F141 undergo large shifts upon idoxifene binding, indicating that the structure of the individual domains is not significantly perturbed in the complex. The aromatic rings of F68 and F141 directly contact the $\varepsilon$ methyl groups of M71 and M144, and the combined chemical shift perturbations of these residues indicate a local disturbance in this region upon idoxifene binding. Overall, it is clear that while side-chain shift changes are localized around the hydrophobic pockets of both domains, backbone shift changes extend to the tether and the rear of the domains.

\subsection{Structure determination}

Intramolecular NOEs within $\mathrm{CaM}$ and within idoxifene and intermolecular NOEs between idoxifene and CaM were quantified using a 2D ${ }^{13} \mathrm{C}-{ }^{15} \mathrm{~N}$-double-half-filtered NOESY spectrum of a $2: 1$ idoxifene : CaM solution (Fig. 5), and the identity of protein resonances was confirmed using a $3 \mathrm{D} \omega_{1}$ ${ }^{13} \mathrm{C}-{ }^{15} \mathrm{~N}$-half-filtered ${ }^{13} \mathrm{C}$-edited NOESY-HSQC spectrum. Most of the intermolecular NOEs arising from the phenyl, $p$-iodo-phenyl, $p$-phenoxy, and ethyl groups of the idoxifene (Fig. 1) are to protein side-chain resonances in the vicinity of the two hydrophobic pockets exposed on calcium binding; none are observed to the opposite faces of the CaM domains. However, it is immediately striking that the phenyl, $p$-iodophenyl, $p$-phenoxy, and ethyl groups mostly have substantial NOEs to the same resonances on the protein despite those resonances belonging to nuclei that are distributed widely across each domain (Fig. S3). Such an NOE distribution can 


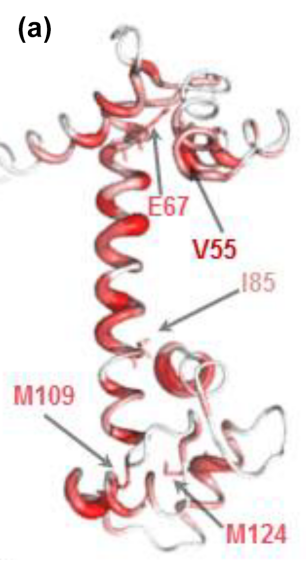

(c)
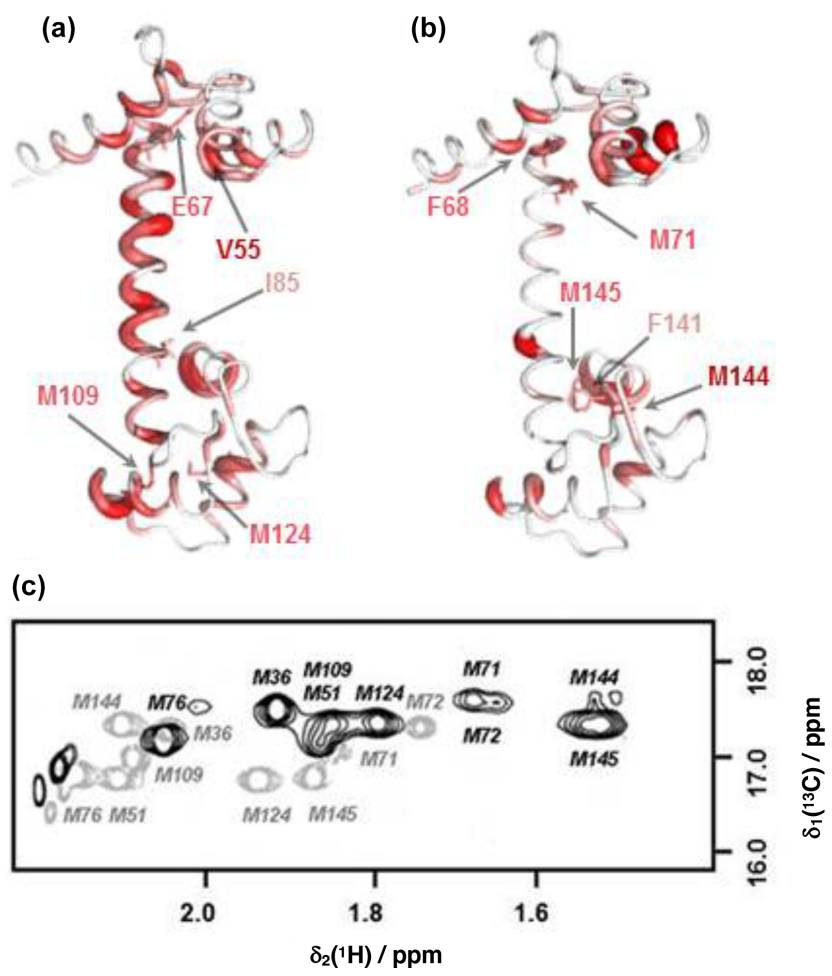

Figure 4. Comparison of backbone and side-chain chemical shift changes in $\mathrm{CaM}$ resonances upon binding idoxifene. (a) and (b): heat map representations of $\mathrm{CaM}$ chemical shift changes upon idoxifene binding, shown for clarity using the coordinates of the unliganded CaM X-ray structure, 1cll. The hydrophobic pocket of the $N$ terminus at the top and the rear of the C-terminal hydrophobic pocket is at the bottom. In (a) residues are coloured according to the weighted average (WA) backbone shift changes observed in the idoxifene : CaM complex, with gradation from white (WA shift $=0.0 \mathrm{ppm}$ ) to red (WA shift $>0.7 \mathrm{ppm}$ ) and the broadest ribbon indicating the maximum shift changes. The locations of M109 and M124 by the hydrophobic pocket of the C domain are indicated. Residues I85 and E67 are indicated, as they define the central portion of the flexible tether joining the two domains. The position of V55, which undergoes a large shift change as in the CaM : M13 complex, is also indicated. In (b) residues are coloured according to the largest side-chain ${ }^{1} \mathrm{H}$ shift changes observed upon idoxifene binding (shifts $>0.50 \mathrm{ppm}$ ), with colour gradation and ribbon width depicted as in (a). The locations of key residues such as three of the nine methionines in the vicinity of the hydrophobic pockets and F68 and F141 are indicated. (c) A 2D ${ }^{13}$ C CT-HSQC spectrum showing the $\varepsilon$ methyl methionine signals of free $\mathrm{CaM}$ (grey) and idoxifenebound CaM (black).

be associated with elevated levels of spin diffusion at extended mixing times, but the mixing time employed here $(100 \mathrm{~ms})$ is the same as or shorter than that used in the structure determination of other CaM-ligand complexes (Craven et al., 1996, Osawa et al., 1998, 1999), where spin diffusion was not significant, and there is no significant difference in rotational correlation times between these complexes, since NOEs over extended distances are not observed. Moreover,

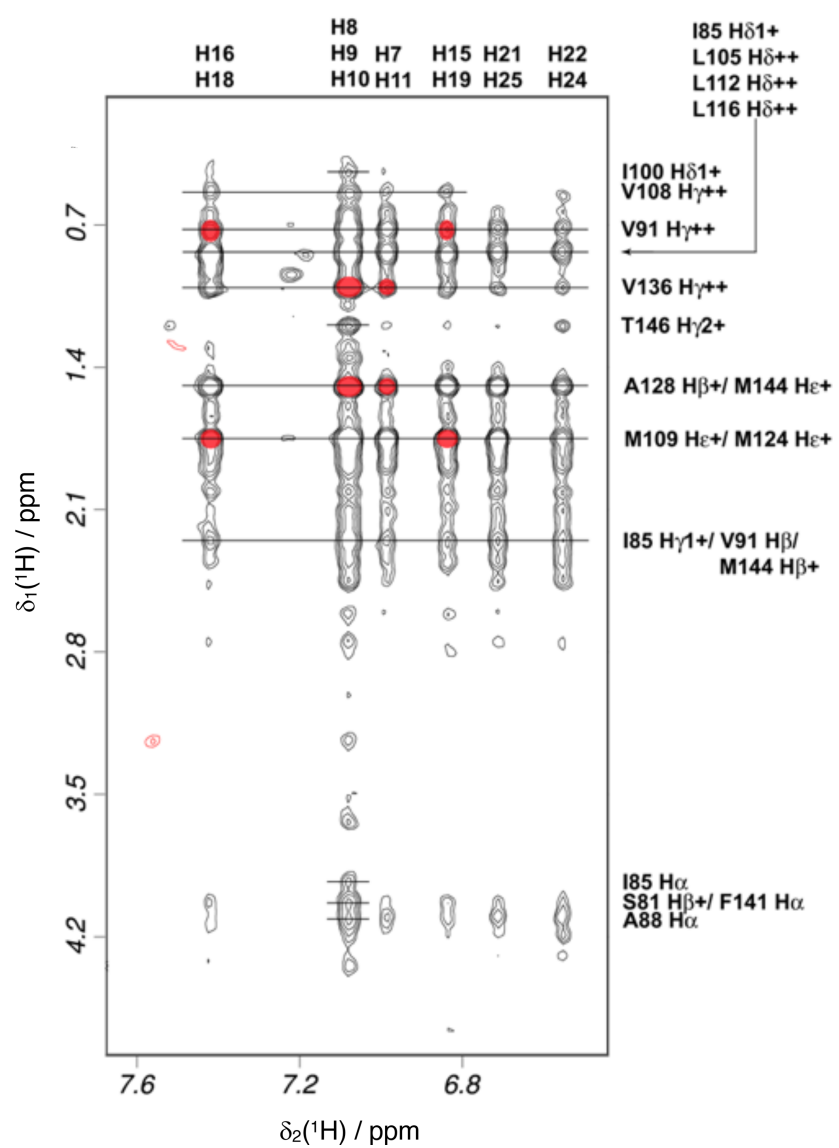

Figure 5. Intermolecular NOEs between idoxifene and CaM. A region of the $2 \mathrm{D}{ }^{13} \mathrm{C}-{ }^{15} \mathrm{~N}$-double-half-filtered NOESY spectrum of the $2: 1$ idoxifene: $\mathrm{CaM}$ complex, processed to exclude the ${ }^{13} \mathrm{C} /{ }^{15} \mathrm{~N}$ bound resonances in the $\omega_{2}$ dimension and to include only the ${ }^{13} \mathrm{C}$ bound resonances in the $\omega_{1}$ dimension. The ligand resonances corresponding to each strip are marked on the top edge as follows: $p$-iodo-phenyl ring (H16/H18), phenyl ring $(\mathrm{H} 8 / \mathrm{H} 9 / \mathrm{H} 10)$, phenyl ring $(\mathrm{H} 7 / \mathrm{H} 11), p$-iodo-phenyl ring (H15/19), and $p$-phenoxy ring (H21/25 and H22/24). Assignments of the resonances from tr $2 \mathrm{c}$ are shown on the right. A red circle over the cross peak indicates examples of NOEs that are violated in the structure calculation corresponding to the data in Fig. S3.

spin-diffusion effects are not dominant between nuclei within the ligand when it is bound in the complex. Hence, spin diffusion alone cannot account for the unusual similarity in intensities of the intermolecular NOEs involving the phenyl, $p$-iodo-phenyl, $p$-phenoxy, and ethyl groups.

Initially, standard NOE-restrained structure calculations were performed including intra-protein, intra-ligand, and intermolecular NOEs, classified as strong, medium, or weak according to intensity (assigned upper bounds of 2.5, 3.5, and $5.0 \AA$ ). The resulting structures did not converge to a well-defined ensemble, and only high-energy structures with widespread NOE violations resulted. Since the conformations of the two individual domains of mammalian $\mathrm{CaM}$ are 
almost invariant across all deposited X-ray structures and the intra-protein NOE distribution within the domains of the $2: 1$ idoxifene : CaM complex reflected closely that of free CaM, focus was shifted to a restrained docking strategy. This allowed the NOE violation energies in the restrained dynamics to be isolated to the intermolecular NOEs rather than be spread widely across the $\mathrm{CaM}$ domains.

In contrast to the invariance of individual domain structures within CaM complexes, the relative position of the two domains varies considerably between deposited structures. NOE-restrained structure calculations where the individual domain structures were fixed but the flexible tether between the two domains was allowed conformational freedom led to closed structures of the idoxifene : CaM complex. However, there was little convergence in the relative position of the two domains, and widespread intermolecular NOE violations remained. Consequently, NOE-restrained docking to a closed structure that well represented the chemical shift changes in the protein backbone (cf. Figs. 3 and S2), that of the CaM : M13 (myosin light-chain kinase peptide) complex, was investigated. With the intermolecular NOEs calibrated directly according to their intensities, the docked structures distributed the idoxifene molecules widely across the hydrophobic surfaces of the two domains of CaM, but 64 of the 180 NOEs remained violated in the lowest-energy structure. When the intermolecular NOEs were all assigned upper bounds of $5.0 \AA$ and the contacting side chains within the individual domains of $\mathrm{CaM}$ were allowed conformational flexibility, the lowest-energy structures converged, whereby the idoxifene molecules occupied one of two sites on $\mathrm{CaM}$ in the vicinity of the hydrophobic pocket on each domain (Fig. 6ab), and the NOE violations in the lowest-energy structures dropped to an average of 5. However, while the docked idoxifene molecules delineated two binding sites in $\mathrm{CaM}$, the orientation of the individual idoxifene molecules was very variable between structures.

In order to establish whether the variability of orientation of the idoxifene ligands in their sites could be reduced, a gradation of upper-bound limits according to intensities of the intermolecular NOEs was re-introduced. Increased deviation from a uniform upper bound of $5.0 \AA$ led to increased restraint violations and, in general, pulled the idoxifene molecules towards the centre of mass of $\mathrm{CaM}$ but without any discernible decrease in the variability of the orientation of individual idoxifene molecules. An alternative approach was thus investigated where the protein in the docking protocol was simplified to one domain - the tr2c domain. This approach removes NOE violation energies that inadvertently pull an idoxifene molecule towards the binding site of the other domain. With the intermolecular NOEs assigned upper bounds of $2.5,3.5$, and $5.0 \AA$, a minimum of 26 of 82 NOEs were violated.

The high proportion of intermolecular NOE violations, coupled with the substantial number of NOEs between distant parts of the ligand and the same resonances on the pro-
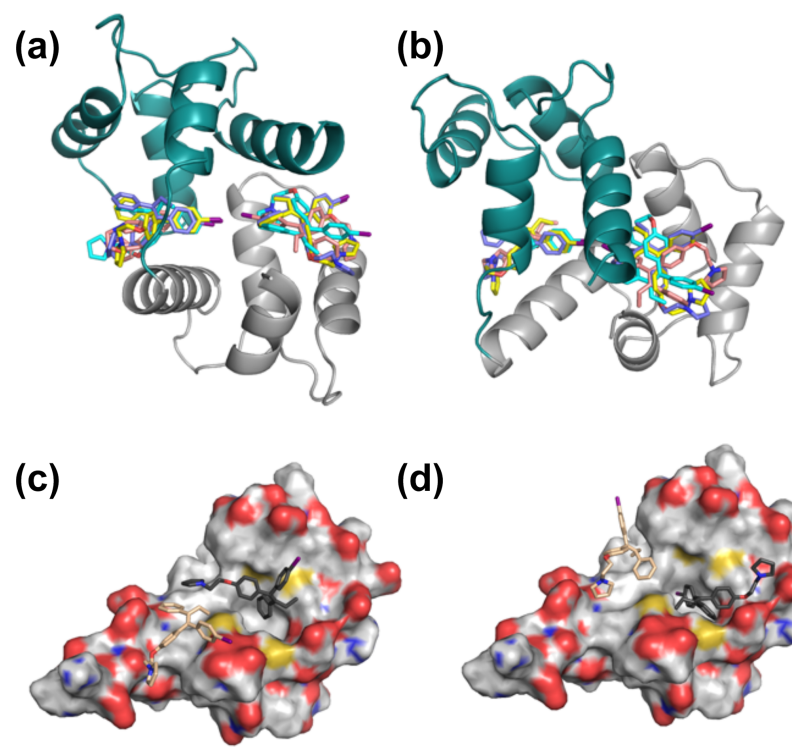

(d)

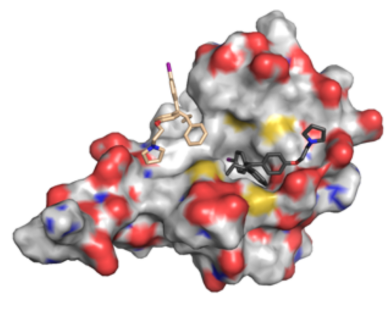

Figure 6. Representative $2: 1$ idoxifene : CaM complex structures. Panels (a) and (b) are orthogonal views of the 2:1 idoxifene: $\mathrm{CaM}$ complex calculated using NOE-restrained docking of two molecules of idoxifene to CaM coordinates based on the CaM : M13 complex (pdb entry 1cdl). In this calculation, the upper bounds of all observed intermolecular NOEs were set to $5 \AA$. CaM domains $\operatorname{tr} 1 \mathrm{c}$ and $\operatorname{tr} 2 \mathrm{c}$ are coloured green and grey respectively and the idoxifene molecules are coloured in pairs (blue, cyan, yellow, or beige) derived from single calculations. View B is orientated to coincide with parts $\mathrm{C}$ and D. Panels (c) and (d) show the positions of four molecules of idoxifene derived from a single NOE-restrained docking calculation to the $\operatorname{tr} 2 \mathrm{c}$ domain of $\mathrm{CaM}$ (from pdb entry 1cll), illustrating one arrangement of four conformations that satisfy the NOE restraints. The four idoxifene molecules are shown in pairs for clarity only. The two darker-coloured idoxifene molecules have either the phenyl group (c) or the $p$-iodo-phenyl group (d) by the pocket in the hydrophobic surface of the domain. The lightercoloured molecules also satisfy NOEs that are within reach of the ligand-binding site associated with the tr1c domain. The surface representation of $\operatorname{tr} 2 \mathrm{c}$ is coloured as follows: carbon (grey), nitrogen (blue), oxygen (red), and sulfur (yellow).

tein (Fig. 5), points to a complex where the ligand undergoes conformational exchange while bound to the protein. In order to simulate this in the NOE-restrained docking, the number of idoxifene molecules included was increased until all of the NOE-assigned upper bounds of $2.5,3.5$, and $5.0 \AA$ were satisfied. No interaction potentials were included between idoxifene molecules in order that multiple binding conformations could be satisfied simultaneously in the calculations, where ligands occupied the same space. With the introduction of four idoxifene molecules, all of the NOE restraints could be satisfied. A representative structure with no NOE violations is shown in Fig. $6 \mathrm{c}-\mathrm{d}$. The NOEs are satisfied by idoxifene placing either the $p$-iodo-phenyl (Fig. 6c) or phenyl group (Fig. 6d) by the pocket in the hydrophobic surface of the domain and orienting the $p$-phenoxy and ethyl groups alter- 
nately towards or away from the position that would be occupied by the tr1c domain (cf. Fig. $6 c-d$ and b). The two other idoxifene molecules also satisfy NOEs that are within reach of the ligand-binding site associated with the tr1c domain (left-hand side of Fig. 6b). When this calculation is repeated with full-length $\mathrm{CaM}$ (in the closed form derived from the CaM : M13 complex), the NOE restraints were satisfied using four idoxifene molecules per domain, and the positions of the idoxifene molecules in the full-length complex followed the pattern observed in the binding to tr2c, where either the $p$-iodo-phenyl group or phenyl group occupies the mouth of the hydrophobic pocket and the $p$-phenoxy and ethyl groups lie along the hydrophobic surface in one of two directions (Fig. 6).

\section{Discussion}

There is now a considerable body of data on CaM-ligand interactions, including solution and solid-state studies on the interactions with both peptides and small molecules (Meador et al., 1992; Ikura et al., 1992; Vandonselaar et al., 1994; Craven et al., 1996; Osawa et al., 1998; Harmat et al., 2000; Horvath et al., 2005; Kovesi et al., 2008). CaM-peptide interactions are normally satisfactorily described by a single set of coordinates for the ligand, representing an average structure, and this is reflected in the good agreement between the structure of these complexes determined using X-ray and NMR spectroscopy methods (Ikura et al., 1991; Meador et al., 1992; Ikura et al., 1992; Maximciuc et al., 2006). However, this approach is less appropriate in the case of many CaMsmall ligand interactions. For example, two DPD : CaM complex structures show substantial differences in the orientation of the ligand (Fig. S4b) and extent of CaM domain closure, while computation of the same complex suggests that $\mathrm{CaM}$ adopts a more globular structure in solution relative to the conformations observed in the X-ray structures (Harmat et al., 2000; Kovesi et al., 2008). Other anomalies also often appear in CaM-small ligand complexes. For example, while $\mathrm{X}$-ray structures of TFP : CaM complexes have been solved with one, two, and four TFPs bound to CaM, NMR measurements and computation show conclusively that the principal binding mode in solution involves two TFP molecules bound with indistinguishable affinity, one to the equivalent site in each domain (Vandonselaar et al., 1994; Craven et al., 1996). Both the above scenarios illustrate that $\mathrm{CaM}-$ small ligand complexes normally require a description including a combination of stoichiometry, relative affinity, exchange dynamics, and structure.

\subsection{Binding affinity and stoichiometry}

The principal binding mode of idoxifene to $\mathrm{CaM}$ has a stoichiometry of $2: 1$ (according to both fluorescence and NMR measurements) and a dissociation constant of $\sim 300 \mathrm{nM}$, resulting from a diffusion-controlled on-rate of $\sim 10^{8} \mathrm{M}^{-1} \mathrm{~s}^{-1}$ and a ligand dissociation rate of $\sim 30 \mathrm{~s}^{-1}$. This dissociation constant indicates that idoxifene has at least an order of magnitude stronger affinity for CaM than that observed for most other antagonists, with the exception of calmidazolium $\left(K_{d} \sim 1-10 \mathrm{nM}\right)$, DPD $\left(K_{d} \sim 18 \mathrm{nM}\right)$, and other bifunctional ligands (Reid et al., 1990; Harmat et al., 2000; Trevitt et al., 2005). The $K_{d}$ value determined here is lower than the previously reported $\mathrm{IC}_{50}$ value for idoxifene, $1.5 \mu \mathrm{M}$ (Hardcastle et al., 1996), which was derived indirectly from the inhibition of calmodulin-dependent cyclic AMP phosphodiesterase and under experimental conditions different from here. On addition of more than two equivalents of idoxifene, further small chemical shift changes are observed for some of the CaM resonances (e.g. K13 and N137 in Fig. 2) in the fast exchange regime on the NMR timescale. Such behaviour has been observed also for other small ligands binding to CaM, such as W-7, J-8, and TFP (Craven et al., 1996; Osawa et al., 1998), and is attributed to secondary, weaker binding phenomena.

\subsection{Domain closure}

The evidence points to $\mathrm{CaM}$ closing to a compact, globular structure on binding idoxifene. The observed distribution and magnitude of backbone chemical shift changes in the $2: 1$ idoxifene : CaM complex are closely similar to those on formation of the CaM : M13 complex, which forms a tightly closed, compact conformation (Ikura et al., 1991; Barbato et al., 1992). In CaM complexes in which the ligand binds to only one of the two domains and the complex does not close, the chemical shift changes in the vast majority of the amide protons in the central linker are very small, usually less than $0.1 \mathrm{ppm}$ (Elshorst et al., 1999). No direct NOEs were observed between the two domains of CaM in the $2: 1$ idoxifene : CaM complex, but this was also the case for the W-7: CaM complex, which forms a tightly closed conformation similar to that of the CaM : M13 complex (Osawa et al., 1998, 1999). Indeed, the direction and magnitude of the backbone proton chemical shift changes for residues in the flexible linker (e.g. E82 and D80) in the W-7 : CaM complex are similar to those observed on formation of the $2: 1$ idoxifene : CaM complex (cf. Fig. S2 and Fig. 6 in Osawa et al., 1999). Moreover, the backbone chemical shift changes within the central tether in the idoxifene : CaM complex cannot be attributed solely to proximity to idoxifene - the intermolecular NOEs and the largest side-chain chemical shift changes are not located in the tether (Fig. $4 a-b$ ). In addition, backbone chemical shift changes for T110-L112 are indicative of the formation of an $\alpha$ helical structure, while those of M144-T146 are indicative of a loss of $\alpha$ helical structure, as reported for the CaM : M13 complex (Ikura et al., 1991). Finally, in the NOE-restrained docking calculations, when the residues in the flexible tether region were allowed conformational freedom, the lowest-energy structures were always closed. 


\subsection{Ligand distribution}

The distribution of NOEs between CaM and idoxifene resonances reflects closely the distribution of the largest chemical shift changes in protein side-chain resonances observed upon idoxifene binding. The contributing side chains are located around the hydrophobic pockets of both CaM domains, and no large chemical shift changes are observed for side chains in the tether or the rear of the domains (Fig. 4b). The strongest intermolecular NOEs were to the $\varepsilon$ methyl groups of methionine residues, and these resonances undergo the largest chemical shift changes (Fig. 4c). Substantial upfield chemical shift changes in these methionine resonances have been reported for numerous peptide and ligand complexes of $\mathrm{CaM}$ and attributed to ring current effects from the aromatic groups of the bound ligand. It is well established that the high abundance of methionines is essential in the biological function of $\mathrm{CaM}$, the side chains of which can provide the conformational variability that enables the binding of a wide range of ligands (Osawa et al., 1998; Elshorst et al., 1999).

The lack of substantial chemical shift perturbations in the phenylalanine rings in the vicinity of the hydrophobic pockets and the low number of NOEs observed between phenylalanine residues and idoxifene indicate that the ligand does not occupy the hydrophobic pockets fully (Fig. 4b). Only F68 and F141 significantly change chemical shift on idoxifene binding, and these changes reflect the loss of $\alpha$ helical structure in the M71-A73 and M144-T146 regions that is typically seen on peptide binding (Ikura et al., 1991). A likely driver for this loss of $\alpha$ helix is that the unwinding allows the methyl group of, for example, T146 to join the hydrophobic surface that interacts with the ligand. This interpretation is supported by the observation of strong NOEs between the hydrophobic groups of idoxifene and T146 $\gamma$ and the chemical shift changes for F141 and M144, which contact each other in the tr2c domain (and F68 and M71 in the equivalent positions in the tr1c domain). Strong NOEs and large chemical shift changes were observed for other hydrophobic residues located over the surface around the hydrophobic pockets, such as I85 and A128 (Figs. 3 and 4).

Overall, the distribution of intermolecular NOEs in the $2: 1$ idoxifene : CaM complex is unusual (Fig. 5) in that each of the substituents around the central double bond of idoxifene (Fig. 1) appears to be close to the same atoms of CaM despite the size and relative rigidity of the propeller structure in this part of the ligand. Moreover, the atoms on $\mathrm{CaM}$ that are close to each of these substituents of idoxifene are widely distributed across the hydrophobic surfaces of the domains (Fig. S3). The unusual distribution of NOEs is not the result of spin diffusion and therefore must reflect some positional heterogeneity within the complex. As free and bound $\mathrm{CaM}$ is in the slow exchange regime on the NMR timescale and only a single set of resonances is observed for the $2: 1$ idoxifene : CaM complex, idoxifene must exchange between these positions while bound to $\mathrm{CaM}$; i.e. the exchange rate between sites must be significantly faster than the dissociation rate. This interpretation is supported by the failure of standard structure determination methods to converge to a single conformation for the complex and by NOE-restrained docking calculations being unable to satisfy the NOEs when only one idoxifene molecule per CaM domain was present in the calculation. When the upper bounds of the NOE restraints were relaxed, the calculated structures delineated the two binding sites in the proteins, which matched well with the chemical shift perturbations observed on idoxifene binding. However, the orientation of the idoxifene molecules was not defined by these calculations, and the introduction of less conservative upper bounds required multiple orientations of idoxifene to be satisfied.

Hence, the relatively even distribution of intermolecular NOEs (Fig. 5) is only readily explicable in terms of multiple binding orientations for the two idoxifene molecules and establishes that the complex cannot be described by a single structure. In the NOE-restrained docking, a minimum of four orientations of idoxifene per $\mathrm{CaM}$ domain were required to satisfy the NOEs. The positions and orientations of the four idoxifene molecules still varied between calculations but can be described approximately by a combination of four conformers (Fig. 6), two where the $p$-iodo-phenyl group occupies the mouth of the hydrophobic pocket and the $p$-phenoxy group lies in the direction of either helix 1 or helix 7 and two where the phenyl group occupies the mouth of the hydrophobic pocket and the $p$-phenoxy group has one or another of the above orientations. More precise positioning of idoxifene molecules is not readily obtained from the NOE-restrained docking calculations for several reasons. Firstly, the relative position and orientation of the tr $1 \mathrm{c}$ and tr2c domains may differ slightly from those in the CaM : M13 complex, and this is not readily determinable without multiple NOEs between the domains. Secondly, the conversion of NOE cross-peak intensities to distances is not unambiguous, as an accurate measurement of the relative populations of the conformers is not independently available. Thirdly, although four idoxifene molecules per domain satisfy the NOE restraints, this is a minimum number of conformers rather than a uniquely determined number.

\subsection{Differences with previous models}

The positionally dynamic mode of binding of idoxifene to $\mathrm{CaM}$ contrasts with the common view that small ligands bind to proteins in a single orientation when the binding affinity is sub-micromolar. However, this paradigm has been challenged by a number of studies (Chattopadhyaya et al., 1992; Carroll et al., 2011; Hughes et al., 2012) that show dynamic binding modes not unrelated to the behaviour of idoxifene when bound to CaM. Indeed, the retention and redistribution of ps- $\mu$ s dynamic modes within protein residues in complexes involving a range of drugs have been shown to favour high-affinity binding in a multidrug transcription repressor 
(Takeuchi et al., 2014). There are two main differences between the behaviour of idoxifene and previously determined CaM-small ligand complexes: the requirement for a broad ensemble rather than a well-positioned bound ligand and the degree of occupancy of the hydrophobic pockets. The requirement for multiple orientations of idoxifene to describe the complex was, however, proposed previously on the basis of molecular modelling (Edwards et al., 1992). Although there are some differences in the conformations and orientations of the bound idoxifene molecules, in both this and an earlier computational model, the aromatic groups of the ligand are not located deep in the hydrophobic pockets, in contrast to the behaviour of the W-7, J-8, and DPD : CaM complexes (Fig. S4). Furthermore, based on the results of the molecular modelling and subsequent SARs of idoxifene analogues, it was suggested that the binding of idoxifene to $\mathrm{CaM}$ would produce a compact globular protein structure similar to that observed in the peptide and TFP complexes (Hardcastle et al., 1996).

\subsection{Role of iodine}

In the molecular modelling study, the increased $\mathrm{CaM}$ antagonism of idoxifene relative to tamoxifen was attributed to the presence of a hydrophobic group such as iodine (Edwards et al., 1992). However, in subsequent SAR studies it was found that substitution of the iodine with a more hydrophobic group such as a butyl chain did not improve $\mathrm{CaM}$ antagonism (Hardcastle et al., 1995). Similarly, J-8, an analogue of W-7 with iodine in place of chlorine, was also found to be the most potent $\mathrm{CaM}$ antagonist in a series of derivatives bearing different halogens in the naphthalene rings (MacNeil et al., 1988). These data and the observation that in the J-8: CaM complex the iodine is placed inside the hydrophobic pockets of the CaM domains led to the conclusion that the affinity for $\mathrm{CaM}$ correlates with the van der Waals radius of the halogen (Craven et al., 1996). However, in the idoxifene : CaM complex, the iodine is not positioned deeply inside the hydrophobic pockets (Fig. 6c-d) but contacts the sulfur atoms and methyl groups of M109 and M144 and the oxygen of E127. Similar interactions are observed between the chlorine in the ligand Kar-2 and M109 and E114 in the X-ray structure of the Kar2 : CaM complex (Horvath et al., 2005). In addition, Kar-2 shows a mode of binding similar to that of idoxifene: both ligands bind to the hydrophobic surface of the domains but not to residues located deep in the hydrophobic pockets. This mode of binding is also observed for $\mathrm{CaM}$ in a complex with a peptide derived from the myristoylated alanine-rich C kinase substrate (MARCKS) and with a peptide derived from the HIV-1 matrix protein p17 (Yamauchi et al., 2003; Izumi et al., 2008). The X-ray structure of the CaM : MARCKS complex shows that the terminal lysine side chains of the peptide are disordered. The presence of multiple conformers is suggested to keep the peptide flex- ible to maximize contacts with the acidic residues located over the surfaces of the two domains.

\subsection{Role of charge}

Electrostatic interactions have previously been proposed to be important in the binding of small ligands to $\mathrm{CaM}$; for example, W-7, J-8, DPD, TFP, and tamoxifen (Edwards et al., 1992; Vandonselaar et al., 1994; Craven et al., 1996; Osawa et al., 1998; Harmat et al., 2000) all contain a flexible chain connected to a basic nitrogen (Figs. 1 and Fig.4SC). A similar picture emerges in the idoxifene : CaM complex. The pyrrolidine ring appears to occupy multiple areas of the protein surface located around the pockets where it can contact the glutamic acid residues; intermolecular NOEs from the pyrrolidine ring to the glutamate side chains of both domains indicate their close proximity. The two X-ray structures of $\mathrm{CaM}$ complexed with DPD, which has a propeller structure similar to that of idoxifene, also show multiple orientations of the analogous basic side chain (Fig. S4b). In some idoxifene : CaM conformers, the pyrrolidine ring is oriented toward the flexible tether that connects the two domains, bringing the pyrrolidine nitrogen close to E84 and E87. A similar orientation of the basic chain is observed in the solution structure of the W-7 : CaM complex (Fig. S4c) and also proposed in the computational model of the $\mathrm{CaM}$ : idoxifene complex (Edwards et al., 1992).

\section{Conclusion}

The observation that four idoxifene molecules are sufficient to satisfy the NOE restraints in the closed form of full-length $\mathrm{CaM}$ argues strongly that the $2: 1$ complex is an ensemble where each idoxifene molecule is predominantly in the vicinity of one of the two hydrophobic patches, fluctuating between a conformational distribution. The $\mathrm{CaM}$ molecule is predominantly in the form where the $\mathrm{N}$ - and $\mathrm{C}$-terminal domains are in close proximity, meaning that the idoxifene molecules are able to contact both domains simultaneously. In addition, these results show that the substantial occupation of the hydrophobic pocket observed with TFP, J-8, W-7, and DPD does not appear to be an essential component of highaffinity binding. This is further supported by the observation that there are other $\mathrm{CaM}$ complexes with high-affinity ligands that do not bind into the hydrophobic pockets. It also explains the results of many SARs on idoxifene: synthetic modification of the aromatic rings has not led to substantial improvement in CaM antagonism. The model presented here opens up opportunities to design substantially higher-affinity antagonists of CaM activity. In addition to extending the repertoire of $\mathrm{CaM}$ antagonism, the dynamic mode of binding adds to the growing number of similar binding modes reported recently for small-molecule ligands. 
Data availability. The underlying research data can be accessed from the University of Sheffield ORDA database (https://doi.org/ 10.15131/shef.data.15113511, Milanesi et al., 2021)

Supplement. The supplement related to this article is available online at: https://doi.org/10.5194/mr-2-629-2021-supplement.

Author contributions. LM, CRT, and BW performed most of the experimental work. AMH, ST, and LLPH provided expert support in NMR methodology and data analysis. LM, BW, CAH, and JPW prepared the manuscript with contributions from all the co-authors. The project was initiated by CAH and JPW.

Competing interests. The authors declare that they have no conflict of interest.

Disclaimer. Publisher's note: Copernicus Publications remains neutral with regard to jurisdictional claims in published maps and institutional affiliations.

Special issue statement. This article is part of the special issue "Robert Kaptein Festschrift". It is not associated with a conference.

Acknowledgements. We thank Stephen Neidle for providing the idoxifene and Jeremy Craven and Svetlana Sedelnikova for expert technical assistance.

Financial support. This research has been supported by the Biotechnology and Biological Sciences Research Council (grant nos. E15611 and S007965) and the Wellcome Trust (grant no. 047301).

Review statement. This paper was edited by Sebastian Hiller and reviewed by Walter Chazin and one anonymous referee.

\section{References}

Barbato, G., Ikura, M., Kay, L. E., Pastor, R. W., and Bax, A.: Backbone dynamics of calmodulin studied by N-15 relaxation using inverse detected 2-dimensional NMR-spectroscopy - the central helix is flexible, Biochemistry, 31, 5269-5278, https://doi.org/10.1021/bi00138a005, 1992.

Brown, S. C., Weber, P. L., and Mueller, L.: Toward complete H-1-NMR spectra in proteins. J. Magn. Reson., 77, 166-169, https://doi.org/10.1016/0022-2364(88)90042-x, 1988.

Brünger, A. T.: X-PLOR. Version 3.1: A system for X-ray crystallography and NMR, Yale University Press, New Haven, CT, USA, 1992.
Byer, S. J., Eckert, J. M., Brossier, N. M., Clodfelder-Miller, B. J., Turk, A. N., Carroll, A. J., Kappes, J. C., Zinn, K. R., Prasain, J. K., and Carroll, S. L.: Tamoxifen inhibits malignant peripheral nerve sheath tumor growth in an estrogen receptor-independent manner, Neuro-Oncology, 13, 28-41, https://doi.org/10.1093/neuonc/noq146, 2011.

Carroll, M. J., Gromova, A. V., Miller, K. R., Tang, H., Wang, X. S., Tripathy, A., Singleton, S. F., Collins, E. J., and Lee, A. L.: Direct detection of structurally resolved dynamics in a multiconformation receptor-ligand complex, J. Am. Chem. Soc., 133, 64226428, https://doi.org/10.1021/ja2005253, 2011.

Chattopadhyaya, R., Meador, W. E., Means, A. R., and Quiocho, F. A.: Calmodulin structure refined at 1.7 Angstrom resolution, J. Mol. Biol., 228, 1177-1192, https://doi.org/10.1016/00222836(92)90324-D, 1992.

Chou, J. J., Li, S. P., Klee, C. B., and Bax, A.: Solution structure of $\mathrm{Ca} 2+$-calmodulin reveals flexible hand-like properties of its domains, Nat. Struct. Biol., 8, 990-997, https://doi.org/10.3410/f.1002399.26607, 2001.

Cifuentes, E., Mataraza, J. M., Yoshida, B. A., Menon, M., Sacks, D. B., Barrack, E. R., and Reddy, G. P. V.: Physical and functional interaction of androgen receptor with calmodulin in prostate cancer cells, P. Natl. Acad. Sci. USA, 101, 464-469, https://doi.org/10.1073/pnas.0307161101, 2004.

Craven, C. J., Whitehead, B., Jones, S. K. A., Thulin, E., Blackburn, G. M., and Waltho, J. P.: Complexes formed between calmodulin and the antagonists J-8 and TFP in solution, Biochemistry, 35, 10287-10299, https://doi.org/10.1021/bi9605043, 1996.

Dolan, K., Montgomery, S., Buchheit, B., DiDone, L., Wellington, M., and Krysan, D. J.: Antifungal activity of tamoxifen: In vitro and in vivo activities and mechanistic characterization, Antimicrob. Agents Ch., 53, 3337-3346, https://doi.org/10.1128/aac.01564-08, 2009.

Dowsett, M., Dixon, J. M., Horgan, K., Salter, J., Hills, M., and Harvey, E.: Antiproliferative effects of idoxifene in a placebocontrolled trial in primary human breast cancer, Clin. Cancer Res., 6, 2260-2267, 2000.

Edwards, K. J., Laughton, C. A., and Neidle, S.: A molecular modeling study of the interactions between the antiestrogen drug tamoxifen and several derivatives, and the calciumbinding protein calmodulin, J. Med. Chem., 35, 2753-2761, https://doi.org/10.1021/jm00093a006, 1992.

Elshorst, B., Hennig, M., Forsterling, H., Diener, A., Maurer, M., Schulte, P., Schwalbe, H., Griesinger, C., Krebs, J., Schmid, H., Vorherr, T., and Carafoli, E.: NMR solution structure of a complex of calmodulin with a binding peptide of the $\mathrm{Ca}^{2+}$ pump, Biochemistry, 38, 12320-12332, https://doi.org/10.1021/bi9908235, 1999.

Finn, B. E., Evenas, J., Drakenberg, T., Waltho, J. P., Thulin, E., and Forsen, S.: Calcium-induced structural-changes and domain autonomy in calmodulin, Nat. Struct. Biol., 2, 777-783, https://doi.org/10.1038/nsb0995-777, 1995.

Frederick, K. K., Marlow, M. S., Valentine, K. G., and Wand, A. J.: Conformational entropy in molecular recognition by proteins, Nature, 448, 325-329, https://doi.org/10.1038/nature05959, 2007.

Gallo, D., Jacquot, Y., Laurent, G., and Leclercq, G.: Calmodulin, a regulatory partner of the estrogen receptor alpha 
in breast cancer cells, Mol. Cell. Endocrinol., 291, 20-26, https://doi.org/10.1016/j.mce.2008.04.011, 2008.

Gulino, A., Barrera, G., Vacca, A., Farina, A., Ferretti, C., Screpanti, I., Dianzani, M. U., and Frati, L.: Calmodulin antagonism and growth-inhibiting activity of triphenylethylene antiestrogens in Mcf-7 human-breast cancer-cells, Cancer Research, 46, 6274-6278, 1986.

Hardcastle, I. R., Rowlands, M. G., Houghton, J., Parr, I. B., Potter, G. A., Jarman, M., Edwards, K. J., Laughton, C. A., Trent, J. O., and Neidle, S.: Rationally designed analogs of tamoxifen with improved calmodulin antagonism, J. Med. Chem., 38, 241-248, https://doi.org/10.1021/jm00002a005, 1995.

Hardcastle, I. R., Rowlands, M. G., Grimshaw, R. M., Houghton, J., Jarman, M., Sharff, A., and Neidle, S.: Homologs of idoxifene: Variation of estrogen receptor binding and calmodulin antagonism with chain length, J. Med. Chem., 39, 999-1004, https://doi.org/10.1021/jm9505472, 1996.

Harmat, V., Bocskei, Z., Naray-Szabo, G., Bata, I., Csutor, A. S., Hermecz, I., Aranyi, P., Szabo, B., Liliom, K., Vertessy, B., and Ovadi, J.: A new potent calmodulin antagonist with arylalkylamine structure: Crystallographic, spectroscopic and functional studies, J. Mol. Biol., 297, 747-755, https://doi.org/10.1006/jmbi.2000.3607, 2000.

Horvath, I., Harmat, V., Perczel, A., Palfi, V., Nyitray, L., Nagy, A., Hlavanda, E., Naray-Szabo, G., and Ovadi, J.: The structure of the complex of calmodulin with KAR2 - A novel mode of binding explains the unique pharmacology of the drug, J. Biol. Chem., 280, 8266-8274, https://doi.org/10.1074/jbc.m410353200, 2005.

Hughes, T. S., Chalmers, M. J., Novick, S., Kuruvilla, D. S., Ra Chang, M., Kamenecka, T. M., Rance, M., Johnson, B. A., Burris, T. P., Griffin, P. R., and Kojetin, D. J.: Ligand and receptor dynamics contribute to the mechanism of graded PPAR gamma agonism, Structure, 20, 139-150, https://doi.org/10.1016/j.str.2011.10.018, 2012.

Ikura, M. and Ames, J. B.: Genetic polymorphism and protein conformational plasticity in the calmodulin superfamily: Two ways to promote multifunctionality, P. Natl. Acad. Sci. USA, 103, 1159-1164, https://doi.org/10.1073/pnas.0508640103, 2006.

Ikura, M., Kay, L. E., Krinks, M., and Bax, A.: Triple-resonance multidimensional NMR-study of calmodulin complexed with the binding domain of skeletal-muscle myosin light-chain kinase Indication of a conformational change in the central helix, Biochemistry, 30, 5498-5504, https://doi.org/10.1021/bi00236a024, 1991.

Ikura, M., Clore, G. M., Gronenborn, A. M., Zhu, G., Klee, C. B., and Bax, A.: Solution structure of a calmodulin-target peptide complex by multidimensional NMR, Science, 256, 632-638, https://doi.org/10.1126/science.1585175, 1992.

Izumi, Y., Watanabe, H., Watanabe, N., Aoyama, A., Jinbo, Y., and Hayashi, N.: Solution X-ray scattering reveals a novel structure of calmodulin complexed with a binding domain peptide from the HIV-1 matrix protein p17, Biochemistry, 47, 7158-7166, https://doi.org/10.1021/bi702416b, 2008.

Kovesi, I., Menyhard, D. K., Laberge, M., and Fidy, J.: Interaction of antagonists with calmodulin: Insights from molecular dynamics simulations, J. Med. Chem., 51, 3081-3093, https://doi.org/10.1021/jm701406e, 2008.
Kuboniwa, H., Tjandra, N., Grzesiek, S., Ren, H., Klee, C. B., and Bax, A.: Solution structure of calcium-free calmodulin, Nat. Struct. Biol., 2, 768-776, https://doi.org/10.1038/nsb0995-768, 1995.

Li, L. and Sacks, D. B.: Functional interactions between calmodulin and estrogen receptor-alpha, Cell Signal., 19, 439-443, https://doi.org/10.1016/j.cellsig.2006.08.018, 2007.

Linse, S., Helmersson, A., and Forsen, S.: Calcium-binding to calmodulin and its globular domains, J. Biol. Chem., 266, 80508054, 1991.

MacGregor, J. I. and Jordan V. C.: Basic guide to the mechanisms of antiestrogen action, Pharmacol. Rev., 50, 151-196, 1998.

MacNeil, S., Griffin, M., Cooke, A. M., Pettett, N. J., Dawson, R. A., Owen, R., and Blackburn, G. M.: Calmodulin antagonists of improved potency and specificity for use in the study of calmodulin biochemistry, Biochem. Pharmacol., 37, 1717-1723, https://doi.org/10.1016/0006-2952(88)90434-0, 1988.

Marshall, E.: Tamoxifen - 'A big deal', but a complex hand to play, Science, 280, 196-196, https://doi.org/10.1126/science.280.5361.196b, 1998.

Maximciuc, A. A., Putkey, J. A., Shamoo, Y., and MacKenzie, K. R.: Complex of calmodulin with a ryanodine receptor target reveals a novel, flexible binding mode, Structure, 14, 1547-1556, https://doi.org/10.1016/j.str.2006.08.011, 2006.

McConnell, H. M.: Reaction rates by Nuclear Magnetic Resonance, J. Chem. Phys., 28, 430-431, 1958.

Meador, W. E., Means, A. R., and Quiocho, F. A.: Target enzyme recognition by calmodulin - 2.4-Angstrom structure of a calmodulin-peptide complex, Science, 257, 1251-1255, https://doi.org/10.1126/science.1519061, 1992.

Milanesi, L., Trevitt, C. R., Whitehead, B., Hounslow, A. M., Tomas, S., Hosszu, L. L. P., Hunter, C. A., and Waltho, J. P.: Idoxifene:Calmodulin complex structures, ORDA [data set], https://doi.org/10.15131/shef.data.15113511, 2021.

Nilges, M.: Calculation of protein structures with ambiguous distance restraints - Automated assignment of ambiguous NOE crosspeaks and disulfide connectivities, J. Mol. Biol., 245, 645660, https://doi.org/10.1006/jmbi.1994.0053, 1995.

Osawa, M., Swindells, M. B., Tanikawa, J., Tanaka, T., Mase, T., Furuya, T., and Ikura, M.: Solution structure of calmodulin-W-7 complex: The basis of diversity in molecular recognition, J. Mol. Biol., 276, 165-176, https://doi.org/10.1006/jmbi.1997.1524, 1998.

Osawa, M., Kuwamoto, S., Izumi, Y., Yap, K. L., Ikura, M., Shibanuma, T., Yokokura, H., Hidaka, H., and Matsushima, N.: Evidence for calmodulin inter-domain compaction in solution induced by W-7 binding, FEBS Lett., 442, 173-177, https://doi.org/10.1016/s0014-5793(98)01637-8, 1999.

Pawar, P., Ma, L. P., Byon, C. H., Liu, H., Ahn, E. Y., Jhala, N., Arnoletti, J. P., McDonald, J. M., and Chen, Y. B.: Molecular mechanisms of tamoxifen therapy for cholangiocarcinoma: Role of calmodulin, Clin. Cancer Res., 15, 1288-1296, https://doi.org/10.1158/1078-0432.ccr-08-1150, 2009.

Powles, T. J.: Extended adjuvant tamoxifen for breast cancer-a new era?, Lancet, 381, 782-783, https://doi.org/10.1016/s01406736(12)62038-8, 2013.

Prozialeck, W. C. and Weiss, B.: Inhibition of calmodulin by phenothiazines and related drugs - Structure-Activity-Relationships, J. Pharmacol. Exp. Ther., 222, 509-516, 1982. 
Reid, D. G., Maclachlan, L. K., Gajjar, K., Voyle, M., King, R. J., and England, P. J.: A Proton nuclear-magnetic-resonance and molecular modeling study of calmidazolium (R24571) binding to calmodulin and skeletal-muscle troponin-C, J. Biol. Chem., 265, 9744-9753, https://doi.org/10.1016/S0021-9258(19)387344, 1990.

Samal, A. B., Ghanam, R. H., Fernandez, T. F., Monroe, E. B., and Saad, J. S.: NMR, biophysical, and biochemical studies reveal the minimal calmodulin binding domain of the HIV-1 matrix protein, J. Biol. Chem., 286, 33533-33543, https://doi.org/10.1074/jbc.m111.273623, 2011.

Shiau, A. K., Barstad, D., Loria, P. M., Cheng, L., Kushner, P. J., Agard, D. A., and Greene, G. L.: The structural basis of estrogen receptor/coactivator recognition and the antagonism of this interaction by tamoxifen, Cell, 95, 927-937, https://doi.org/10.1016/s0092-8674(00)81717-1, 1998.

Swulius, M. T. and Waxham, M. N.: $\mathrm{Ca}^{2+} /$ calmodulindependent protein kinases, Cell. Mol. Life Sci., 65, 2637-2657, https://doi.org/10.1007/s00018-008-8086-2, 2008.

Takeuchi, K., Tokunaga, Y., Imai, M., Takahashi, H., and Shimada, I.: Dynamic multidrug recognition by multidrug transcriptional repressor LmrR, Sci. Rep., 4, 6922, https://doi.org/10.1038/srep06922, 2014.

Trevitt, C. R., Craven, C. J., Milanesi, L., Syson, K., Mattinen, M. L., Perkins, J., Annila, A., Hunter, C. A., and Waltho, J. P.: Enhanced ligand affinity for receptors in which components of the binding site are independently mobile, Chem. Biol., 12, 89-97, https://doi.org/10.1016/j.chembiol.2004.11.007, 2005.
Vandonselaar, M., Hickie, R. A., Quail, J. W., and Delbaere, L. T. J.: Trifluoperazine-induced conformational change in $\mathrm{Ca}^{2+}$-calmodulin, Nat. Struct. Biol., 1, 795-801, https://doi.org/10.1038/nsb1194-795, 1994.

Vogel, H. J., Lindahl, L., and Thulin, E.: Calcium-dependent hydrophobic interaction chromatography of calmodulin, troponinC and their proteolytic fragments, FEBS Lett., 157, 241-246, https://doi.org/10.1016/0014-5793(83)80554-7, 1983.

Wishart, D. S., Bigam, C. G., Yao, J., Abildgaard, F., Dyson, H. J., Oldfield, E., Markley, J. L., and Sykes, B. D.: ${ }^{1} \mathrm{H},{ }^{13} \mathrm{C}$ and ${ }^{15} \mathrm{~N}$ chemical-shift referencing in biomolecular NMR, J. Biomol. NMR., 6, 135-140, https://doi.org/10.1007/bf00211777, 1995.

Yamauchi, E., Nakatsu, T., Matsubara, M., Kato, H., and Taniguchi, H.: Crystal structure of a MARCKS peptide containing the calmodulin-binding domain in complex with $\mathrm{Ca}^{2+}$-calmodulin, Nat. Struct. Biol., 10, 226-231, https://doi.org/10.1038/nsb900, 2003.

Zhang, M., Tanaka, T., and Ikura, M.: Calcium-induced conformational transition revealed by the solution structure of apo calmodulin, Nat. Struct. Biol., 2, 758-767, https://doi.org/10.1038/nsb0995-758, 1995. 